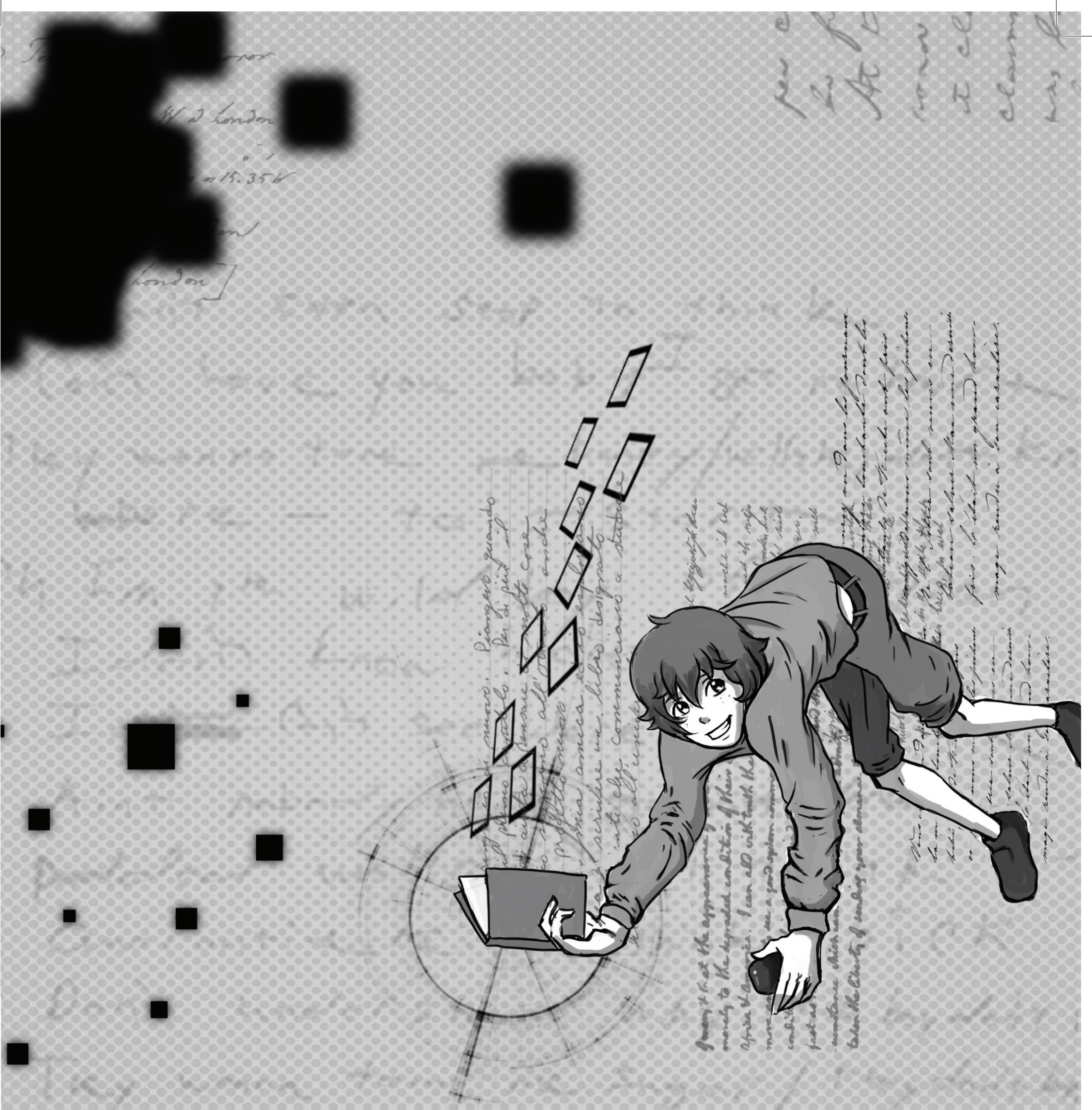




\section{TIC, desarrollo y educación: sociedades en transformación y paradigmas de cambio en Bolivia}

\author{
ICT, development and education: societies \\ in transformation and paradigms of change \\ in Bolivia
}

\author{
Pablo Andrés Rivero Morales (Bolivia) \\ payorivero@gmail.com \\ Universidad Nuestra Señora de La Paz
}

\author{
TIC, desenvolvimento e educação: socie- \\ dades em transformação e paradigmas de \\ câmbio na Bolivia.
}

Politólogo de la Universidad Nuestra Señora de La Paz (Bolivia) y Máster (con distinción) en Comunicación Política Internacional, Advocacy y Campañas de Kingston University (Inglaterra). Ha sido facilitador del programa Focuss. Info de la Facultad de Ciencias Sociales de la Universidad de Rotterdam (Países Bajos) y gestor de Comunidades Virtuales en el Programa de las Naciones Unidas para el Desarrollo (PNUD). Es docente e investigador en la Universidad Nuestra Señora de La Paz (Bolivia) y profesor en la Facultad de Comunicación de la Universidad Riga Stradins (Letonia).

\section{Resumen}

A partir de un marco situacional, donde se describen los cambios socioeconómicos y sociodemográficos importantes en el tejido social boliviano -que por supuesto no son ajenos a las trasformaciones en el resto de Latinoamérica- este artículo indaga sobre las condiciones sociales, demográficas y políticas para la gestión de la tecnología y la información, de manera progresiva y sostenible en la educación; particularmente enfocándose en la educación superior, con el fin de incidir en un masivo acceso y aprovechamiento de la información y, así, contribuir a la genuina democratización para que se reduzcan las asimetrías y se contribuya al desarrollo económico sostenible en Bolivia.

La adopción institucional de principios colaborativos, la generación de "infoestructuras" y la gestación de una "educación libre", a partir del desarrollo tecnológico, son tres propuestas que este artículo ofrece para comprender un nuevo paradigma educativo que genere sinergia hacia: 1) la innovación y la creación de modelos innovadores de negocio, 2) el desarrollo tecnológico propio, mayores y mejores niveles de democratización de la información; y 3) el desarrollo sustentable en el tránsito hacia "la sociedad del conocimiento".

\section{Abstract}

The development of new technologies has always been part of human history, however technologies of information and communication (ICT) emerge dramatically these days becoming determining and mediator factors of human relations at all scales. This radical transformation poses difficulties and great challenges on the educative process.

Starting from a situational framework, this article inquires about the social, demographic and political conditions, for a progressive and sustainable technology and information management in education. This framework describes the

\section{Resumo}

A partir de um marco situacional, onde se descrevem os câmbios socioeconômicos e sociodemográficos importantes no tecido social boliviano -que por suposto não são alheios às transformações no resto de Latino América- este artigo indaga sobre as condições sociais, demográficas e políticas para a gestão da tecnologia e a informação, de maneira progressiva e sustentável na educação; particularmente focando-se na educação superior, com o fim de incidir em um massivo acesso e aproveitamento da informação e, assim, contribuir à genuína democratização para que se reduzam as assimetrias 
important socio-economical and socio-demographic changes in the Bolivian social tissue, which have been obviously affected by the transformations in the rest of Latin America. Focusing on superior education and with the aim to have an impact on the massive access and better use of information therefore contributing to democratization and reducing asymmetry, it can contribute to attain a sustainable economic development in Bolivia.

The adoption of collaborative principles, the development of infostructures and the birth of a "free education" based on new technological development are three of the proposals offered by this article to understand the new educational paradigm. This paradigm must create synergy towards 1.Innovation and creation of innovative business models, 2. Self- technological development, better and higher information democratization levels and 3. sustainable development during the transition to Knowledge Society.

Palabras clave: educación, desarrollo, tecnologías, transformaciones, colaboración, libre, TIC

Palavras chave: educação, desenvolvimento, tecnologias, transformações, colaboração, livre, TIC

\section{Cita recomendada}

Rivero, P . (2012). TIC, desarrollo y educación: sociedades en transformación y paradigmas de cambio en Bolivia. Panorama, 6 (11), $47-64$ e se contribua ao desenvolvimento econômico sustentável na Bolívia.

A adoção institucional de princípios colaborativos, a geração de "info-estruturas" e a gestação de uma "educação livre", a partir do desenvolvimento tecnológico, são três propostas que este artigo oferece para compreender um novo paradigma educativo que gere sinergia para: 1) a inovação e a criação de modelos inovadores de negócio, 2) o desenvolvimento tecnológico próprio, maiores e melhores níveis de democratização da informação; e 3) o desenvolvimento sustentável no trânsito para "a sociedade do conhecimento".

Key Words: education, development, technologies, transformations, collaboration, free, ICT. 


\section{INTRODUCCIÓN}

El desarrollo de las tecnologías hacen parte de la historia de la humanidad. Desde la antigüedad, en todas las culturas y regiones del planeta, los seres humanos han inventado, adaptado, copiado y reproducido una infinidad de herramientas para mejorar las condiciones de vida de colectivos e individuos.

En el pasado reciente, sin embargo, las tecnologías de la información y la comunicación (TIC) emergieron explosivamente en un fenómeno denominado "revolución tecnológica" de las comunicaciones, incorporándose como factores determinantes en la mediación de las relaciones humanas a toda escala, donde la educación se sitúa en una posición de gran impacto y también de grandes desafíos (Sunkel, et al. 2001).

Existen numerosos ejemplos sobre los beneficios de incorporar las TIC a diferentes sectores. Por ejemplo, al desarrollo productivo, a la reproducción del intercambio económico, al diálogo y ejercicio democrático, a la multiplicación de espacios, cada vez, más veloces y canales de acceso e intercambio cultural, etc.

Sin embargo el desarrollo tecnológico para la información y la comunicación en América Latina, y más aún en los países más pobres de la Región, es incipiente. Somos, en definitiva, consumidores pasivos y asumimos elevados costos de transferencia de tecnología producida desde la vanguardia del capitalismo global.

En este escenario, este artículo indaga sobre cuáles son las condiciones sociales, demográficas y políticas para la gestión de la tecnología y la información de manera progresiva y sostenible, fijando la mirada en el campo educativo, que además incida en un masivo acceso y aprovechamiento de la información y contribuya a la genuina democratización, reduzca las asimetrías y contribuya al desarrollo económico sostenible en Bolivia.

El texto fija un horizonte de análisis a partir del marco situacional de la educación y las TIC; y propone algunos puntos de reflexión para los cambios necesarios en el proceso de aprendizaje así como también nuevos paradigmas en la reproducción de información en el ámbito académico.

Se asume desde un inicio que las TIC no son la panacea del desarrollo, la equidad, la nueva era de la comunicación ni tampoco el único camino privilegiado hacia la educación de calidad, pero son una realidad social resultante de procesos y relaciones sociales globalizantes y con un horizonte de visibilidad dado por la "sociedad de la información” postindustrial.

\section{Tecnologías, "globalización" y sociedad de la información}

Este análisis, aborda críticamente los cambios y dinámicas sociales que determinan las nuevas tecnologías de intercambio de información y comunicación, afectando el relacionamiento en la sociedad, proceso que está 
impactando decididamente en el contexto sociopolítico y cultural de lo nacional, lo global y también lo local, pero ante todo condicionando la transformación de las formas de acceso y reproducción del conocimiento.

Así, el punto de partida es la globalización que aparece como el proceso contemporáneo dominante en la comprensión de las relaciones sociales y de intercambio. Este término, que goza de una amplia cantidad de definiciones, enfoques multidisciplinarios y por ello también debates y desacuerdos, se entiende como un "[proceso] político, tecnológico y cultural, así como económico, que ha sido influenciado, sobre todo, por la evolución de los sistemas de comunicación, se remonta sólo a finales de 1960" (Giddens, 1999).

En el mismo sentido, John Tomlinson (1996), observa al proceso de globalización como algo reciente de las últimas tres o cuatro décadas, y lo define como "un proceso de desarrollo veloz de complejas interconexiones entre sociedades, culturas, instituciones e individuos en todo el mundo. Se trata de un proceso social que implica una compresión del tiempo y el espacio, la reducción de las distancias a través de una reducción dramática en el tiempo -ya sea física o representacionalmente- para atravesarlo, por lo que el mundo parezca más pequeño y en cierto sentido, trayendo seres humanos más cerca unos de otros" (citado en Ya’u, 2005 traducción del autor).

Conviene prestar cuidadosa atención a la resignificación del tiempo y el espacio, la velocidad del flujo de información -que incluso ya se expresa en la transmisión en tiempo real-, además de la redefinición de lo "territorial" frente a lo "virtual" como significantes que resultan más potentes, comparado con la mera internacionalización de la economía o las dinámicas sociales y culturales.

Un elemento decisivo para este proceso de globalización, de acuerdo con Giddens, o por ejemplo, Manuel Castells, es el desarrollo tecnológico. Eso sí, hay que considerarlo decisivo más no exclusivo, partiendo de que este desarrollo tecnológico es ante todo un resultado histórico y un hecho jamás aislado de las dinámicas sociales.

Castells (2009) incluso ha desarrollado en la última década una amplia argumentación respecto a la "sociedad de las redes" (Network Society), donde la construcción social del tiempo y el espacio es distinta a la de la sociedad industrial. La ruptura de la secuencia lineal del pasado, presente y futuro; así como la compresión del tiempo, son dos características evidentes de esta nueva "sociedad", donde además las relaciones de poder han cambiado, haciendo un énfasis en la comunicación.

La tecnología, de acuerdo con Irma van der Ploeg, es el resultado de procesos y elecciones sociales, que además están en un continuo "rehacerse". "Las 'tecnologías' en sí mismas no funcionan, las tecnologías son siempre elementos operativos en una práctica particular que está también determinada por otros elementos” (Van der Ploeg, 2003, traducción del autor).

El concepto de tecnología, en tanto proceso y resultado de elecciones sociales, va a ser abordado en este artículo desde la perspectiva de las tecnologías de la información y la comunicación. Debido a su condición de constante evolución y amplio sentido, no hay una definición genérica de las tecnologías de la información y la 
comunicación (TIC). En consecuencia, las TIC podrían ser explicadas a partir de sus tres componentes: información, comunicación y tecnología.

Así, las TIC son tecnologías que permiten el almacenamiento, la producción, reproducción, transmisión, recuperación, recepción y distribución de la información. En las dinámicas comunicacionales latinoamericanas, se complementan y, en algún caso, yuxtaponen tecnologías analógicas y también digitales resultando un complejo mapa de difusión, accesos e interacción.

Sin embargo, el componente digital es el punto de inflexión en el desarrollo tecnológico porque permite a los usuarios, en distintos grados de experticia, editar y combinar textos, imágenes, videos y registros de todos ellos transformados por algoritmos matemáticos que son leídos a través de un software en la computadora. Este es un cambio de lo físico a lo matemático, de fuentes tangibles de información a espacios virtuales (Van Loon, 2008).

Las redes de telecomunicación, las redes informáticas de computadoras interconectadas y las formas de transmisión masiva de convergencia son nuevas tecnologías de transmisión de datos y almacenamiento, basadas fundamentalmente en la fibra óptica, comunicaciones por satélite y avanzados software (Castells, 2009).

Esto que puede parecer ciencia ficción es una realidad comunicacional que está cambiando, en algunos entornos sociales, económicos y culturales más velozmente que en otros. Las formas de intercambio, acumulación, uso y apropiación de recursos informacionales tanto en países desarrollados como en aquellos en vías de desarrollo, incluso dentro de nuestra misma Región (el proceso en Brasil y Chile, no es comparable al que viven Guatemala o Bolivia, por citar algunos ejemplos).

El uso de la información, como señala Liliana Herrera, está transformando las formas de producción, pasando de un paradigma industrial a uno informacional, donde la sociedad se caracteriza por el incremento en el uso de la información como materia prima para producir riqueza (Herrera, 2005).

Este proceso histórico/social se define como la sociedad de la información, que se caracteriza por ser un "Nuevo sistema tecnológico, económico y social. Una economía en la que el incremento de productividad no depende del incremento cuantitativo de los factores de producción (capital, trabajo, recursos naturales), sino de la aplicación de conocimientos e información a la gestión, producción y distribución, tanto en los procesos como en los productos" (Castells, 2004 en Herrera, 2005).

Es decir, el nuevo paradigma de flujo y acceso a la información no es únicamente un proceso de cambio tecnológico informacional/comunicacional, sino que tiene directa implicancia en una nueva economía de la información. Este nuevo paradigma, por tanto, impacta en las formas de generar riqueza y acumulación, lo que tiene directa conexión y trascendencia en el proceso de globalización del capitalismo. 
En ese orden, el sociólogo francés Alain Touraine (2000) sugiere que el capitalismo informacional, en una doble acción, genera excedente que produce desarrollo pero también acentúa las asimetrías y desigualdades lo que produce pobreza. Esto se convierte inmediatamente en un desafío para los gobiernos que deben equilibrar sus políticas de organización y redistribución económica y social con las dinámicas del sistema económico internacional - sobre todo el financiero - (Touraine 2000), resultando así una nueva forma de economía política de la información directamente relacionada a las posibilidades de desarrollo de los países.

La contextualización conceptual antes presentada permite abordar empíricamente la observación sobre las transformaciones y desafíos en la relación de la educación y las TIC en Bolivia, un país en el extremo periférico del acceso a la información y la tecnología, pero que al mismo tiempo se encuentra en un periodo de cambios sociodemográficos, económicos y políticos que se analizan a continuación.

\section{Cambios sociodemográficos, económicos y políticos frente a las perspectivas educativas en Bolivia}

Desarrollo y educación son procesos intrínsecamente relacionados. Es más, las condiciones de posibilidad de lo primero están directamente relacionadas a lo segundo. De hecho, se evidencia que las tres reformas educativas que vivió Bolivia en las últimas seis décadas (1955 Código de la Educación de la Revolución Nacional, la reforma educativa de 1994 -denostada hoy como "neoliberal"-, y la reforma educativa de 2010, cuyo principal horizonte es la "descolonización”), están en sintonía con los paradigmas de desarrollo propuestos desde el poder político.

Sin embargo, es un imperativo comprender que Bolivia fue a lo largo de su historia republicana un país eminentemente agrario. La economía fue históricamente dependiente de la minería -incluso hoy continúa siéndolo en gran medida- y más recientemente de la producción gasífera.

La composición social del país supone que hay una mayoría indígena, aunque esta presunción hasta hace poco inequívoca está siendo materia de debate sociológico, antropológico y metodológico. La categoría "indígena" se emplea desde la perspectiva de la Constitución de 2009 para referirse a los pueblos que habitaban el actual territorio boliviano antes de la llegada española (1540 aprox.). El último Censo Nacional de Población (2001) incluyó una pregunta sobre pertenencia étnica consultando a la población si se consideraban pertenecientes a un grupo indígena. El 62\% de la población mayor de 15 años dijo pertenecer a uno de los grupos étnicos. Sin embargo, no debe darse por concedida y plena la conceptualización absolutista, otras recolecciones de datos muestran, cómo un estudio del PAPEP en 2010, demostró que el 73\% de la población se dice mestiza, dejando así una brecha para la indagación respecto a una relación de pertenencia o filiación a lo indígena (Rivero, 2011a).

A pesar de esta auto-identificación o pertenencia étnica, el tejido social y las relaciones de intercambio están sufriendo en los últimos años transformaciones radicales, sobre todo a partir de la composición demográfica: la población es ahora mayoritariamente urbana (más del 65\%), y además estamos entrando en una curva demográfica histórica donde la mayoría de la población pasa de la niñez y adolescencia a la juventud (FAO, 2007). 
Ambos fenómenos sociodemográficos tienen directa incidencia en la recomposición de un tejido social con características distintas a anteriores décadas, donde las formas de interrelación e intercambio económico, la manera que se accede y consume información, etc. serán necesariamente diferentes. Estas prácticas inciden directamente en el horizonte de expectativas y formas culturales donde se proyectan los individuos y las comunidades en el Estado, el mercado y la sociedad.

Pero las configuraciones de poder están también sufriendo transformaciones inéditas en los últimos años. La Constitución de 2009 y un enorme nuevo marco normativo impulsado por el gobierno de Evo Morales buscan refrendar la reconfiguración del mapa político-institucional de Bolivia, a partir de un ambicioso, contradictorio y conflictivo modelo donde "conviven" autonomías locales, departamentales e indígenas. Como resultado, las instancias y mecanismos de participación y decisión políticas, así como también de diseño de políticas públicas, están siendo transformados en el Estado.

No es casual que todos estos procesos de transformación política, económica y sociodemográfica brevemente descritos anteriormente coincidan también en un momento histórico de transformación de las relaciones sociales a partir de las TIC y emerjan nuevos parámetros culturales. Dichos cambios, por supuesto, no están exentos de tensiones y rupturas sociales y políticas, aunque también coincidan con las formas de acceso, uso y aprovechamiento de la información en un nuevo paradigma de relacionamiento social, donde la tecnología va penetrando e intermediando cada vez más la composición de las redes sociales y los lazos culturales, lo que impacta directamente en la educación, los procesos de aprendizaje, el acceso y manejo de la información.

Por ejemplo, en promedio 7,4 de cada 10 ciudadanos en Bolivia tiene un dispositivo móvil activado. Asimismo, se estima que entre el 38\% y 42\% de la población urbana tiene algún grado de conectividad a internet, mientras que apenas el 5\% de los hogares tiene conexión domiciliaria a internet (Rivero, 2011b). No se han encontrado estudios concluyentes para Bolivia, aunque a partir de las dinámicas en la Región y el resto del mundo, no es descabellado suponer que mucha de esta población con acceso mayor a las nuevas TIC, sea joven.

A partir de estas consideraciones panorámicas y situacionales, se propone indagar acerca de los paradigmas y estrategias, así como también analizar los desafíos que enfrenta la educación para ajustarse en el incontenible flujo de información - sobre todo digital -,continuar motivando procesos cognitivo-reflexivos en las y los estudiantes, así como generar sinergia con la orientación del Estado, el mercado y la sociedad para desarrollar modelos innovadores de negocio, desarrollo tecnológico, una mayor democratización de la información y el desarrollo sustentable.

En ese orden, conviene complementar el panorama antes descrito con algunos datos socioeconómicos que además permitan contextualizar el escenario situacional de la educación en Bolivia. Sin embargo, como se verá, los escasos datos están ante todo orientados a la educación primaria y secundaria, mientras la educación superior formal -objeto de estudio en esta investigación- carece de fuentes estadísticas que acompañe la precisión de estas afirmaciones. 
De los 10 millones de ciudadanos que viven en Bolivia, el 60,1\% vive sobre o por debajo de la línea de la pobreza. Asimismo, el 37,7\% del total de la población vive aún en la pobreza extrema1 (INE, 2011). Este panorama sitúa al país entre los más pobres de América Latina y el Caribe, además de estar lejos aún de alcanzar las Metas del Milenio en materia de reducción de la pobreza (véase cuadro 1). A pesar de ello, los análisis comparativos respecto de la situación en décadas pasadas muestran que el país ha avanzado en indicadores relativos al desarrollo humano y las condiciones de vida.

En cuanto a datos en educación para Bolivia, la tasa de alfabetización en adultos alcanza al 91\% (datos al 2008) con 7,43 como promedio de años de estudio (datos al 2001). Asimismo, la tasa de alfabetismo en la población de 15 a 24 años es del 98\% al 2008 y el 22,4\% de la población ha alcanzado algún nivel de educación superior al año 2008 (INE 2011, CEPAL, 2011 y Banco Mundial, 2011). Es previsible que las cifras de escolaridad y promedio de años de estudio hayan aumentado debido a los efectos de programas de alfabetización, como el "Yo sí puedo" y el "Yo también puedo", y también los bonos familiares por asistencia escolar, implementados por el actual gobierno desde el 2007 .

En cuanto a la educación superior, de acuerdo con las estadísticas oficiales, las universidades públicas y privadas tuvieron cerca de 240 mil estudiantes matriculados, egresados y titulados ${ }^{2}$ en 2008, mostrándose la distribución de la matrícula en el gráfico 2. El número de matriculados, egresados y titulados representa el 8\% de la población económicamente activa (19 a 60 años $\left.{ }^{3}\right)$.

No se ha podido acceder a información oficial que determine, por ejemplo, estratos socioeconómicos de los matriculados, egresados y titulados en instituciones de educación superior pública y privada en Bolivia.

Consecuentemente, este panorama general de datos es insuficiente para realizar un análisis sobre la calidad de esa educación ni de los servicios educativos, tanto públicos como privados. Es más, la instancia gubernamental encargada de la medición de la calidad de la educación, el Observatorio Plurinacional de la Calidad Educativa (OPCE), continúa, al momento de redacción de este artículo, en el proceso de gestación institucional; ya que la nueva Ley de Educación promulgada en 2010 continúa en la fase de reglamentación, y por tanto todo el sistema educativo gubernamental aún permanece en "reconstrucción".

En este escenario, Bolivia no cuenta con datos de calidad educativa que puedan ser comparados a nivel regional o global al porque se retiró del sistema de medición de la calidad educativa de la UNESCO y tampoco hace parte del Programme for International Student Assesment (PISA).

1Datos al 2007. El último censo de población realizado en Bolivia fue el año 2001. Un nuevo censo debía realizarse en 2011 aunque el gobierno lo ha postergado hasta el 2012, por lo que, al menos, hasta mediados de 2013 no se contarán con datos actualizados acerca de las características de la población boliviana.

2 Debe hacerse una diferenciación entre egresados y titulados. El tiempo promedio, entre el fin de la currícula (egresado) y la obtención del título académico (titulado), transcurre entre 4 a 9 años, dependiendo de la institución (privada o pública).

3 Hay abordajes que consideran la Población Económicamente Activa (PEA), desde los 14 años, en especial en lo referido al empleo y el subempleo. Sin embargo, este estudio se enfoca en la educación superior, por lo que considera a la PEA desde los 19 años. 
De la misma manera que la escasa disponibilidad de datos sobre educación, la vasta mayoría de literatura y análisis sobre TIC y educación, está orientada a la educación inicial, primaria y secundaria, siendo la educación superior la que menos reflexión y propuestas tiene.

\section{Reflexiones: las TIC y la transformación de la educación superior}

El presente artículo también aborda el escenario boliviano de cambio en los medios y relaciones de intercambio, los cambios en la composición del tejido social, las transformaciones en el acceso y manejo de la información, así como las condiciones adversas de asimetrías y desigualdades hacia la sociedad de la información"

Si se observa el pasado reciente de las instituciones de educación superior en Bolivia, se puede afirmar que éstas llegaron a tener y ejercer el monopolio del acceso al conocimiento. Esta condición, sin embargo, está siendo rebasada en muchas instituciones educativas latinoamericanas, el acceso a la información ya no puede ser controlada y las formas de re/producir conocimiento están cambiando, especialmente por las deficiencias en la concepción, infraestructura y dotación de recursos pedagógicos, pero también -como se discutirá más adelanteporque los enfoques de enseñanza y aprendizaje, así como de desarrollo de competencias y análisis crítico, en la educación superior, están quedando rezagados.

Como punto de inicio de cualquier análisis, no se debe perder de vista el complejo panorama que Latinoamérica vive, siendo la región más desigual del planeta (Peres y Hilbert 2009). Ante ello, María Belén Fernández argumenta que "ciertamente, la institución educativa se enfrenta hoy al desafío de orientar el acceso de los sujetos a la sociedad de la información, de manera más justa y equitativa” (Fernández, 2008).

Fernández (2008) argumenta que:

«el problema del acceso desigual a la tecnología ha sido concebido desde una retórica dominante en términos de subdesarrollo, de atraso de los aparatos o de falta de acceso a Internet, retórica más funcional al mercado internacional que a una verdadera democratización de uso. En cambio, la idea de la brecha digital nos ayuda a pensar en las desigualdades de acceso como reflejo de las desigualdades sociales» ( Ibíd, cursivas del autor).

Existen diversos abordajes al problema de la brecha digital, entendida como la diferencia socioeconómica entre personas, comunidades o estados que utilizan las TIC en la vida cotidiana y aquellas que no tienen acceso a las mismas. Existen algunos modelos analíticos para el abordaje de la brecha digital, como el propuesto por Neil Selwyn 5 que sugiere la apropiación de la tecnología desde la tenencia en el hogar. Sin embargo, al estar sujeto a las condiciones del mercado para la adquisición de equipos y servicios (por ejemplo, computadoras y conexión a internet), cualquier medida correctiva o política pública se enfrenta al panorama general de desigualdad que afecta a

4Conviene aclarar que el horizonte de visibilidad es la sociedad del conocimiento, concepto que aún provoca amplio debate pero que se constituye en una etapa aún distante.

5 Mencionado en Peres y Hilbert 2009, capítulo VIII. 
toda la Región, en particular a Bolivia, Colombia, Guatemala o República Dominicana donde la enorme desigualdad se mantiene o incluso está creciendo (CEPAL, 2010).

En ese orden, si bien la dotación de equipos a centros educativos de educación primaria, secundaria o superior es una medida necesaria e impulsada por diversas organizaciones internacionales, no tiene un decidido impacto en la reducción de la brecha digital en tanto fenómeno socioeconómico. Pero además "es evidente que la "propiedad" de la tecnología no garantiza su uso crítico ni una incorporación adecuada al ámbito educativo. Para generar condiciones de usabilidad está claro que no alcanza con saber usar la computadora, sino que es necesario reflexionar acerca de las posibilidades y limitaciones de las TIC para poder aplicarlas en procesos de desarrollo social de vasto alcance” (Fernández, 2008).

Es más, estudios confiables, entre ellos uno publicado recientemente por el Banco interamericano de Desarrollo (BID), han demostrado que "el sólo hecho de adquirir computadoras y/o ampliar las horas de uso de ellas y de internet (infraestructura y acceso) no incide directamente en un mejor rendimiento académico” (Chong, 2011).

En síntesis, el fierro y los cables no son condiciones per se para el acceso a una mejor información y a procesos educativos de calidad. La facilidad de acceso a grandes volúmenes de información no garantiza un proceso cognitivo-analítico ni el desarrollo de competencias críticas. Ahora bien, esto es excluyente al constatar que las desigualdades en América Latina se están acentuando y creciendo también desde la perspectiva de acceso y uso de las TIC. A pesar de ello, no se puede encarar el problema con medidas que sean "tecnológicamente deterministas."

La brecha digital, asimismo, no se expresa únicamente en datos socioeconómicos o en la propiedad de equipos de acceso y manejo de información. Es también percibida en la cotidianeidad de las aulas en la educación superior cuando los contenidos académicos, los enfoques de dinámicas de aula para la enseñanza y el aprendizaje, así como el desarrollo de competencias y análisis crítico en la educación superior, se enfrentan a la paradoja de la hiperinformación.

Ante ello, María Belén Fernández comenta acertadamente:

Jesús Martín Barbero señalaba que la tecnología remite hoy no a unos aparatos, sino a nuevos modos de percepción y de lenguaje, nuevas sensibilidades y escrituras. Las nuevas tecnologías de la comunicación implican la dispersión de los saberes, el cambio de identidades, la desestabilización de lo que creíamos legítimo (Fernández, 2008).

En ese sentido, las políticas públicas en educación superior, las estrategias en las universidades públicas y privadas, las metodologías y dinámicas en el aula a cargo de los docentes y el equipo de apoyo, las actividades de involucramiento de estudiantes en investigación, innovación y desarrollo, entre otras instancias, deben también cambiar de paradigma y así proyectar nuevas condiciones de posibilidad para la educación de calidad y equidad en el marco de la generación de tecnología e información a gran escala. Por supuesto, estos procesos también le sirven a las empresas y a los proyectos de emprendimiento. 
Las TIC en el proceso educativo debe ser asumida como un medio, no como un fin, incluso si se trabaja en capacitación o desarrollo de destrezas de manejo. Esto incluso para quienes hacen uso intensivo de la misma. Porque, como se afirmó al principio de este artículo, la tecnología es el resultado de procesos y dinámicas sociales que dependen de ellas, y por tanto su generación y desarrollo es, ante todo, un proceso social.

\section{Transformando la educación: tres criterios que orientan el proceso}

Como resultante de la contextualización situacional y análisis de las condiciones dadas en la relación TIC-educación superior, se propone a continuación abordar tres criterios que orienten el proceso de transformación de la educación superior en el contexto de las TIC. Estos criterios, que cierran esta ponencia y abren el debate, no pretenden ser concluyentes ni cerrados, todo lo contrario, como se verá, son líneas que permiten visibilizar de manera real las condiciones de posibilidad de la educación superior en los tiempos por venir.

\section{a. Acciones colaborativas y compartir información}

La acción colaborativa no es un valor ajeno a las sociedades latinoamericanas, sin excluir a otras sociedades en el planeta, por supuesto. Consiste en realizar acciones y conseguir resultados en función de apoyo a las destrezas, capacidades y recursos de unos con otros para un beneficio colectivo.

Si asumimos que la hiperinformación es una realidad de nuestro tiempo y que las desigualdades se siguen acentuando producto de la brecha digital de acceso y manejo de las TIC, entre otros factores socioeconómicos, una de las posibilidades que nos queda es compartir información.

Quienes enfrentan como yo el día a día en las aulas universitarias, podrán dar fe de lo siguiente: en general, resulta inverosímil pensar que la fuente inicial de información de las y los estudiantes hoy sea otra que sitios en internet. Es más, en muchos casos, estas fuentes no son académicas y son más bien plataformas de acceso rápido vía buscadores y con contenidos simplificados.

Las acciones colaborativas deben ser motivadas desde el aula como una etapa superior al trabajo en equipo, reconociendo las condiciones de acceso a la información de los estudiantes. Implica una dinámica más intensa y con mayor compromiso por parte de los docentes. Las acciones colaborativas consisten en procurar que el trabajo, investigación o reflexión de unos esté directamente relacionado con los otros y donde los docentes cumplen un rol de facilitador y de filtro de la información.

Las TIC que permiten la interacción y que tienen un crecimiento exponencial entre las nuevas generaciones, entiéndase las redes sociales en internet, pueden viabilizar esta acción colaborativa. Esto implica llevar la 
información que los docentes quieren compartir con los estudiantes al lugar donde los estudiantes "habitan "páginas de la materia donde se comparte información, debates y reflexiones "fuera del aula", es decir, en la plataforma de la red social, reproduciendo los códigos y lenguajes que allí se crean, con la intención de lograr mayor compromiso.

El objetivo será, por tanto, lograr que los trabajos de investigación o de análisis y reflexión crítica en el aula, se vean enriquecidos por la acción colaborativa ex ante y ex post entre los estudiantes, que colaboran con otros estudiantes, así como docentes que se involucran y se convierten en un actor más, fuera del aula.

Compartir información y construir colaborativamente conocimiento son horizontes de cambio enmarcados en nuevos paradigmas éticos, gestores de nuevas acciones de enseñanza y aprendizaje, pero también reproductores de nuevos valores colaborativos, integrados a una multiplicidad de comunidades de práctica.

\section{b. Infoestructuras}

¿Dadas las limitaciones y brechas de acceso y manejo de tecnologías de información, es posible realizar estas acciones en Bolivia? Considero que sí. Es más, no sólo es posible sino que es un imperativo. Es precisamente en países como Bolivia donde este cambio paradigmático debe operarse porque las fuentes tradicionales de acceso a la información sufren la escasez de recursos: las bibliotecas se actualizan a una lentitud trágica y la disponibilidad de fuentes es muy limitada.

En estas condiciones, transformar las bibliotecas en centros de información multi-recursos es un cambio necesario, espacios de integración de formatos impresos y digitales. Para ello debemos gestar bibliotecas temáticas en línea pero que sean abiertas, es decir, que los estudiantes sean capaces de descargar los archivos para leerlos luego, y que estén diseñadas para que cualquier usuario pueda compartirlas vía correo electrónico, plataformas de interacción o redes sociales. En otras palabras, democratizar el acceso al conocimiento, también de forma digital.

La gestión de redes de interacción e intercambio -entre académicos, entre éstos y los estudiantes, pero también entre académicos, estudiantes y la comunidad, los funcionarios públicos y los hacedores de políticas públicas- es una visión conocida como infoestructuras. Este término, a pesar de no tener aún una definición única y absoluta, se puede entender como el desarrollo de estándares de información, herramientas de gestión y el fomento del acceso a nivel comunitario.

Las infoestructuras son condiciones complementarias a las infraestructuras, pero desde la intangibilidad y exponencial reproducción de información. De hecho, parten de la idea que la información es un recurso renovable y que su explotación, a diferencia de otros, no conduce al agotamiento sino más bien a su reproducción.

$6 \quad$ En la denominada Web 2.0 'habitar digitalmente' significa todas aquellas redes sociales y plataformas donde las y los jóvenes interactúan y pasan considerable tiempo semanal, por ejemplo Facebook, MSN Messenger, y en menor medida Twitter. Un breve estudio en la Universidad Nuestra Señora de La Paz, universidad privada de Bolivia, donde he llevado a cabo con estudiantes de seis distintas facultades, demostró que el 100\% tienen cuentas en Redes Sociales, que el $74 \%$ se conecta a estas redes al menos 4 veces por semana y el $45 \%$ todos los días. 
Las infoestructuras, sin embargo, no son software, estructuras físicas o tampoco instalaciones de fierros y cables, las infoestructuras son ante todo personas que asumen la construcción colaborativa y la redistribución de información a quienes tienen menos acceso. Se busca nuevamente democratizar el acceso al conocimiento a partir de ejercicios y prácticas en espacios abiertos que vayan construyendo redes indexadas de información y que puedan ser accedidas, por otros, en campos de búsqueda abiertos?

\section{c. Caminando hacia la educación "libre"}

Se discute cada vez más la apertura del conocimiento como condición para el desarrollo, pues la gestión de la información se ve intermediada por las tecnologías. Así pues, este debate debe llegar también al vehículo intangible por el cual accedemos a la información: los sistemas operativos y el software.

Países como Bolivia pueden convertirse en generadores de software y sistemas operativos resulta inverosímil en el corto plazo, aunque de hecho hay ejemplos destacables a fuerza de pulmón individuals. Inverosímil, más no imposible, como algunos afirman. Las condiciones de posibilidad pasan por generar las precondiciones de gestación, es decir, asumir medidas desde el gobierno, las instituciones educativas y las empresas que viabilicen las precondiciones.

Una de estas precondiciones es una educación que ensanche las libertades en la sociedad de la información, democratice su acceso y apropiación, y favorezca la cultura abierta. Esta visión es la propuesta por el Software Libre, no sólo como práctica y desarrollo tecnológico, sino como horizonte filosófico.

El "Software libre es el software disponible, gratuito o comercialmente, con las premisas de libertad de instalación; plena utilización; acceso al código fuente; posibilidad de modificaciones/perfeccionamientos para necesidades especíícas; distribución de la forma original o modificada, con o sin costos" (Gobierno Electrónico de Brasil, 2004).

Desarrollado en los últimos 20 años de manera colaborativa en todo el mundo, el Software Libre no es únicamente una opción tecnológica que impulsa la innovación y que propone tejidos industriales y postindustriales que reduzcan asimetrías y favorezcan la libre competencia; también es, desde una perspectiva educativa, un resultado tangible y exitoso del ejercicio colaborativo.

En el caso boliviano, en sintonía con el programa de migración gubernamental brasilero al Software Libre, la recientemente promulgada Ley de Telecomunicaciones y TIC de Bolivia propone un plan de priorización del código abierto que tenga impacto en el gobierno electrónico, sistemas de seguridad del Estado y también educación a partir del Software Libre. Este plan será técnica y estratégicamente viable si las autoridades asumen con decisión la

\footnotetext{
$7 \quad$ Un ejemplo de construcción de infoestructuras es la plataforma Delicious (http://www.delicious.com/).

8 Véase las empresas bolivianas asociadas en la Fundación iFaro (http://www.ifaro.org/).
} 
migración primero al Software libre; y segundo, si se desarrollan condiciones de gestión de gobierno electrónico. Sin embargo, esta visión también debe ser adoptada por las instituciones educativas públicas y privadas.

Comúnmente se infiere que el Software Libre es más económico, o directamente gratuito, y supone una reducción de costos. Eso no es necesariamente cierto. Indagando el tema con la Comunidad del Software libre en Bolivia, se ha reconocido que los costos de desarrollo y mantenimiento pueden llegar a significar sumas importantes. El enfoque por tanto no es de reducción del gasto sino de inversión sostenible, inversión que optimice la generación y creación de recursos informáticos y que logre dinamismos distintos en el Estado, el mercado y la sociedad.

Es decir, en la medida que la educación esté orientada a la generación de tecnología innovadora y basada en la apertura mental y colaborativa, se estará incorporando criterios y valores de transparencia, libertad de pensamiento, generación, innovación y optimización de recursos de la mano de la tecnología en un proceso amplio. Además, se reproducen valores democráticos ya que el Software Libre garantiza la igualdad de oportunidades y posiciona un concepto distinto de seguridad de la información. El software libre, en este orden, es parte de la vanguardia del proceso. 


\section{Referencias bibliográficas}

1. Banco Mundial. (2011). Datos del Banco Mundial. Datos electrónicos, disponibles en http:// datos.bancomundial.org/pais/bolivia (revisado 24/04/2012).

2. Castells, M. (2009). Communication power. Oxford: Oxford University Press.

3. CEPAL. (2010). Panorama social de América Latina 2009. Informe anual de la División de Desarrollo Social y la División de Estadística y Proyecciones Económicas de la CEPAL. Documento online, disponible en http://www.eclac.org/publicaciones/xml/9/37839/PSE2009texto-completo.pdf (revisado en 20/06/2012).

4. CEPAL. (2011). CEPALstat, estádisticas de América Latina y el Caribe. Información online, disponible en http://websie.eclac.cl/infest/ajax/cepalstat.asp?carpeta=contenidos (revisado en 20/08/2011).

5. Chong, A. (Ed.). (2011). Conexiones del desarrollo: Impacto de las nuevas tecnologías de la información. Washington DC: Desarrollo de las Américas - BID.

6. FAO. (2007). La mujer en la agricultura, medio ambiente y la producción rural - Bolivia. Publicación online, disponible en http://www.fao.org/docrep/007/ad927s/ad927s01.htm\#fn2 (revisado en 20/08/2011).

7. Fernández, M. (2008). Las TIC en la Educación: Algunos elementos para el debate. Revista digital Question n. ${ }^{\circ}$ 19, agosto de 2008. Artículo online, disponible en www.perio.unlp.edu.ar/ question (revisado en 04/06/2012).

8. García Zaballos, A. (2011). Interconexión o acceso, ¿qué va antes? En: TICs para el Desarrollo, un blog del Banco Interamericano de Desarrollo. 20 de Julio de 2011. Online, disponible en http://blogs.iadb.org/tics/2011/07/20/interconexion-o-acceso-que-va-antes/ (revisado 26/06/2012).

9. Giddens, A. (1999). Runaway World. Londres: Profile Books.

10. Gobierno Electrónico de Brasil. (2004). Guía Libre Referencia de Migración para Software Libre del Gobierno Federal de Brasil. Documento online del Comité Técnico para Implementación de Software Libre. Disponible en: www.governoeletronico.gov.br/anexos/versaoem-espanhol-do-guia-livre (revisado 16/05/2012).

11. Jenkins, H. (2006). Convergence Culture. New York: New York University Press.

12. Herrera, L. (2005). Contexto sociopolítico de la sociedad de la información. Programa de Maestría en Estudios Políticos. Facultad de Estudios Políticos y Relaciones Internacionales. Bogotá: Pontificia Universidad Javeriana.

13. Instituto Nacional de Estadística. (2011). Estadísticas Sociales. Datos electrónicos, disponibles en http://www.ine.gob.bo/default.aspx (revisado 22/06/2012).

14. Van Loon, J. (2008). Media Technology. Maidenhead: Open University Press. 
15. Mosco, V. (1996). The Political Economy of Communication. Londres: SAGE.

16. Peres, W. y Hilbert, M. (Eds.). (2009). La sociedad de la información en América Latina y el Caribe. Santiago de Chile: Naciones Unidas.

17. Van der Ploeg, I. (2003). Biometrics and Privacy. A note on the politics of theorizing technology. In: Information, Communication \& Society, . 6 (1). 85-104, [online] disponible en http:// www.informaworld.com/smpp/title content=t713699183 (24/06/2012).

18. Rivero, P. (2011a). Coca Política: re/construcción social de la coca como 'artefacto' ritualdiscursivo-político en Bolivia. Ponencia presentada en el VI Congreso de la Asociación de Estudios Bolivianos - AEB. Sucre, Junio 2011.

19. Rivero, P. (2011b). Ley de Telecomunicaciones: alcances, límites, preocupaciones y propuestas a la normativa, con énfasis en Internet. En Cuadernos de Análisis de la Fundación Milenio. La Paz: Fundación Milenio.

20. Sunkel, G., Trucco, D., y Möller, S. (2011). Aprender y enseñar con las tecnologías de la información y las comunicaciones en América Latina: potenciales beneficios. Santiago de Chile: División de Desarrollo Social de la CEPAL. Documento online, disponible en: http://www.eclac.cl/ publicaciones/xml/9/42669/sps-169-tics-aprendizajes.pdf(revisado en 24/06/12).

21. Touraine, A. (2000). ¿Podemos vivir juntos? : Iguales y Diferentes. México DF: Fondo de Cultura Económica.

22. UNESCO. (2009). Medición de las Tecnologías de la Información y la Comunicación (TIC) en Educación. Documento Técnico n. ${ }^{\circ}$ 2. Montreal: Instituto de Estadística de la UNESCO. Versión online, disponible en http://unesdoc.unesco.org/images/0018/001883/188309s.pdf (revisado en 22/06/12).

23. Weinberger, D. (2011). Too big to know.New York: Basic Books.

24. Ya'u, Y. (2005). Globalisation, ICTs, and the New Imperialism: Perspectives on Africa in the Global Electronic Village. Africa Development, Vol. 30, Nos. 1 \& 2, pp. 98-124 [online] disponible en: www.ejisdc.org/ojs2/index.php/ejisdc/article/view/520/254(revisado en 14/05/12). 